\title{
RELACIÓN ENTRE LOS MEDIOS DE DEPÓSITO Y LOS ENSAYOS QUIIMICOS EN LOS CARBONES DE SAMACÁ (BOYACÁ, COLOMBIA)
}

(Relations between the Deposits Means and the Chemical Trials in the Samacá Coal)

Jaime Humberto Suescún Torres

Ingeniería Geológica, Uptc-Sogamoso, marcaf_geo@yahoo.com

(Recibido Septiembre 3 de 2006 y aceptado Marzo 9 de 2007)

\section{Resumen:}

Este estudio sirve de base para otro más regional, que comprende la cartografía geológica, caracterización y cálculo de reservas para los carbones pertenecientes al nivel Ktg-2 - Ktg-3 de la Formación Guaduas. El propósito de la investigación fue establecer la relación entre los análisis químicos y los ambientes de depósito, comparando las columnas estratigráficas con las curvas de los ensayos químicos. Después de describir la columna estratigráfica desde el punto de vista litológico se muestrearon los mantos de los niveles carboníferos para ensayos próximos, últimos, petrográficos y otros, para finalmente hacer comparaciones con los ambientes de depósito mediante tablas y gráficas. De las gráficas comparativas se deduce que a medida que los ambientes se hacen más continentales aumenta el contenido de azufre, ceniza y materia volátil, mientras que los contenidos de vitrinita y poder de reflectancia disminuyen. Se recomienda adelantar estudios sobre un mayor número de muestras que incluyan los demás estudios realizados.

\section{Abstract:}

This is a base for a broader regional study, which comprises the geologic cartography, characterization and calculation of reserves for coals pertaining to the level Ktg-2 - Ktg-3 of the Guaduas Formation. The intention was to establish the relation between the chemical analysis and the deposit environment, by comparing the strata-graphic columns with the curves of the chemical tests. After describing the strata-graphic column from the lito-logical point of view, there were taken samples of the carboniferous mantles levels for further petro-graphic trials. Finally to make comparisons with the environment deposits by means of tables and graphics. From the comparative graphs it is deduced that as the environment become more continental, it increases the content of sulfur, ash and volatile matter, whereas the contents of vitrinite and the power of reflectance diminish. It is recommended to carry on studying on a greater number of samples that include the other studies already made.

Palabras clave: Carbón, Geología, Samacá.

Key words: Coal, Geology, Samacá.

\section{INTRODUCCIÓN}

$\mathbf{E}$ 1 carbón es considerado el recurso mineral energético más abundante del planeta; encontrar la manera de "predecir" su calidad a partir de sus propiedades fisicoquímicas ha demandado diversos esfuerzos.

Desde el punto de vista de su origen, los carbones se forman en turberas, que son ecosistemas donde la tabla de agua está cerca o ligeramente sobre el suelo mineral, por lo que la vegetación asociada produce materia orgánica (turba) a una tasa superior a la que los procesos de degradación pueden descomponer.

Los tipos de turba dependen del aporte de sedimentos, el tipo de línea de costa y la extensión de las aguas continentales dentro del océano.

Las diferentes propiedades químicas dependen directamente de las características ambientales en las cuencas y sus fenómenos asociados. En Samacá (Boyacá) es importante determinar dichas relaciones para establecer con mayor precisión el comportamiento de los mantos de acuerdo con su posición estratigráfica y la acción de eventos posteriores (ver figura 1).

Son muchos los estudios que se han realizado en el área sobre la geología y la calidad de los carbones. Arboleda (1987) estudió los carbones coquizables del área; Pérez et ál. (1987) estudiaron la caracterización de los carbones, y Sarmiento (1992) estudio la estratigrafía y los medios de depósito de la Formación Guaduas.

El propósito de la presente investigación es analizar la génesis de los carbones desde el punto de vista de los ambientes de depósito y correlacionarlos con los resultados de los ensayos químicos con el fin de encontrar afinidades que puedan ser utilizadas como herramientas de exploración.

\section{METODOLOGÍA}

Se realizó la descripción estratigráfica detallada de la Formación Guaduas, sobre el flanco occidental del Sinclinal Checua-Lenguazaque, centrándose en los niveles Ktg-2 y 


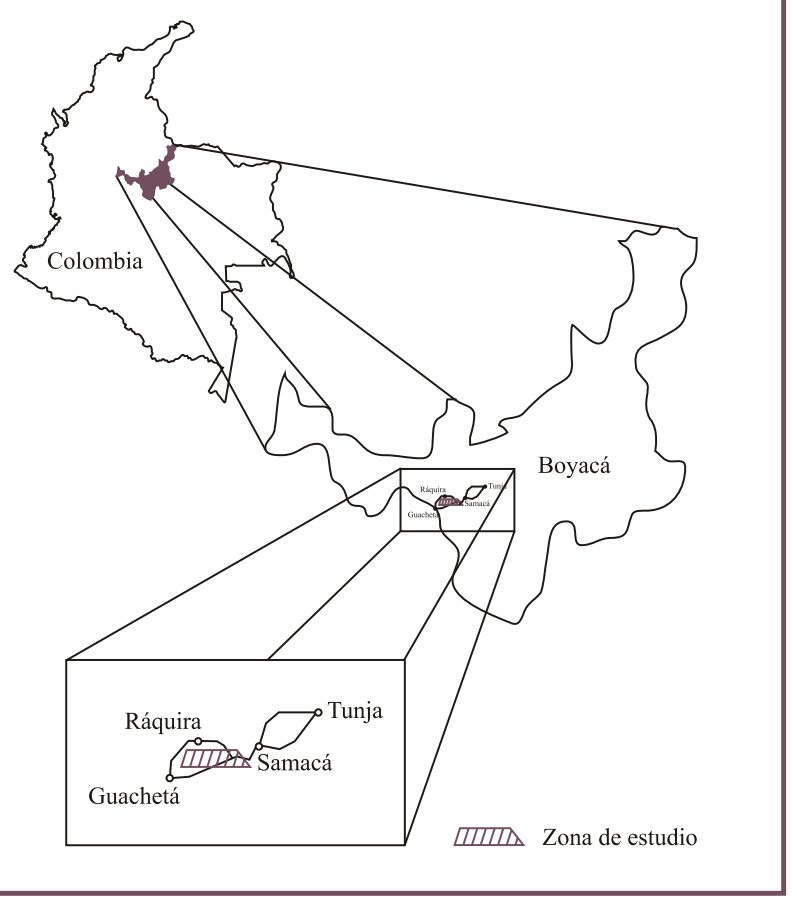

Figura 1. Mapa de

Localización.

Ktg-3, por contener mantos de carbón con interés económico importante.

Los ensayos químicos se realizaron en los laboratorios de la Uptc Sogamoso, teniendo en cuenta las normas ASTM para ensayos de carbón. Para la caracterización de los carbones se realizaron los siguientes ensayos:

- Análisis próximo, incluye: humedad residual, cenizas, materia volátil, carbono fijo.

- Análisis último: azufre total.

- Ensayos varios, incluye: poder calorífico, humedad de equilibrio.

- Análisis de propiedades plásticas: FSI (Free Swelling Index).

\section{- Vitrinita}

En la columna se describió la composición granulométrica, color, estructuras sedimentarias, espesor, geometría y fósiles; adicionalmente se muestrearon los mantos de carbón para análisis de calidad y petrografía, los mantos de carbón se identificaron con la misma nomenclatura utilizada por los mineros de la región (ver figura 2).

Los resultados de los diferentes análisis fueron sometidos a comparaciones para determinar el comportamiento de los diferentes mantos de carbón y su interrelación ambiental (ver tabla 1).

\section{GEOLOGÍA}

La cuenca carbonífera Tausa-Samacá se ubica en la parte central de la Cordillera Oriental (Hubach, 1957); está conformada por rocas sedimentarias de edad Cretácico Superior a Terciario Inferior, y afectada tectónicamente por las fallas de Boyacá y Soapaga, que originan la estructura regional conocida como el sinclinal Checua-Lenguazaque.

\subsection{Estratigrafia regional}

El Grupo Guadalupe de base a techo se divide en Formación Arenisca Dura, Formación Plaeners, Formación Labor y Tierna. La Formación Arenisca Dura no aflora en el sector de Samacá.

La Formación Plaeners presenta una secuencia limolítica silícea con intercalaciones de arcilla y gran contenido de fósiles. La Formación Labor consta de areniscas de grano medio a grueso con intercalación de lutitas fosilíferas (foraminíferos). La Formación Arenisca Tierna presenta areniscas de grano medio a grueso con alta bioturbación y laminación plano paralela continua.

La Formación Guaduas se define como un conjunto de estratos que contienen mantos de carbón, se divide en cinco niveles. Nivel Ktg-1, sucesión de arcillolitas con limolitas grises, caracterizado por no presentar mantos de carbón. Nivel Ktg-2, capas interestratificadas de arenisca de grano fino y arcillolitas negras, caracterizada por presentar los primeros mantos de carbón. Nivel Ktg-3, secuencia de arcillolitas con intercalaciones de lutitas negras carbonosas donde se ubica el mayor número de mantos de carbón. Nivel Ktg-4, areniscas de grano medio a fino con intercalaciones de arcillolitas gris pardo y mantos de carbón de poco espesor (Duarte y Mariño, 2001). Nivel Ktg-5, conjunto de arcillolitas abigarradas caracterizadas por no presentar mantos de carbón.

La Formación Cacho se establece como una secuencia de areniscas cuarzosas de grano medio a conglomerático con intercalaciones de limolitas, presencia de marcas de corriente y paleocanales.

Se observan depósitos de origen glacial, fluvioglacial y aluvial.

\subsection{Estratigrafia local}

Se describen aproximadamente $700 \mathrm{~m}$ (ver figura 2) de la Formación Guaduas en el sector de Samacá, de la siguiente forma:

En el nivel Ktg-1 se levantaron $136 \mathrm{~m}$, constituidos por arcillolitas poco compactas con niveles limolíticos e intercalaciones de areniscas cuarzosas de grano fino, concreciones subdivididas por tabiques, marcas de corriente y estratificación ondulada no paralela. Fue depositado en un ambiente costero intramareal sustentado por la baja bioturbación y alta sedimentación de partículas orgánicas en suspensión. 
En el nivel Ktg-2 se levantaron $152 \mathrm{~m}$ de arcillolitas grises pardas con intercalación de areniscas de grano fino a medio, dentro de las cuales se ubican los primeros mantos de carbón (Cisquera, Tesoro y Tesorito) seguidos por una secuencia homogénea de arcillolitas gris pardo con intercalaciones de lutitas negras carbonosas, presencia del segundo grupo de mantos de carbón (Milagro, Alisos, Gemela, Consuelo, Rubí y Bocatoma). Con ambiente supramareal lacustre se ubican los primeros mantos de carbón entre los niveles arenosos, explicando la transición intramareal-supramareal, aumentando el aporte de materia orgánica a la cuenca. La presencia de arcillolitas y lutitas en la parte superior de la secuencia indica muy bajo aporte de material clástico, favoreciendo la formación de mantos de carbón, ya que las zonas lacustres son medios reductores sin cambios fuertes en las facies deposicionales. El nivel Ktg-2 termina con $15 \mathrm{~m}$ de arenisca cuarzosa con acuñamientos y marcas de corriente, intercalaciones de lutitas grises amarillas. Ambientes de depósito con ríos, desbordes, canales meandriformes y algo lacustres explican las variaciones granulométricas y la presencia de materiales finos en periodos de anegación.

En el nivel Ktg-3 se levantaron $200 \mathrm{~m}$ de arcillolitas con intercalaciones de areniscas gradadas hacia la base, presencia de un manto de carbón (La Cisquera Segunda), seguido por una secuencia de arenisca gris parda con flasser de arcillolitas negras carbonosas y algunas capas de lutita. Se observa el tercer grupo de mantos de carbón (La Limpia, Piedro, Veta Grande Siete Bancos). Posteriormente se observa una secuencia de arcillolita gris parda con flasser de lutitas negras carbonosas y el último manto explotable (La Vidriosa). Predominan ambientes de pantanos, canales estuarinos, llanuras bajas, ríos meandriformes y zonas intermareales que explican las grandes variaciones granulométricas, estratificación flasser y mantos de carbón de gran espesor; $13 \mathrm{~m}$ de arenisca cuarzosa de grano fino a medio, subredondeada, bien seleccionadas,

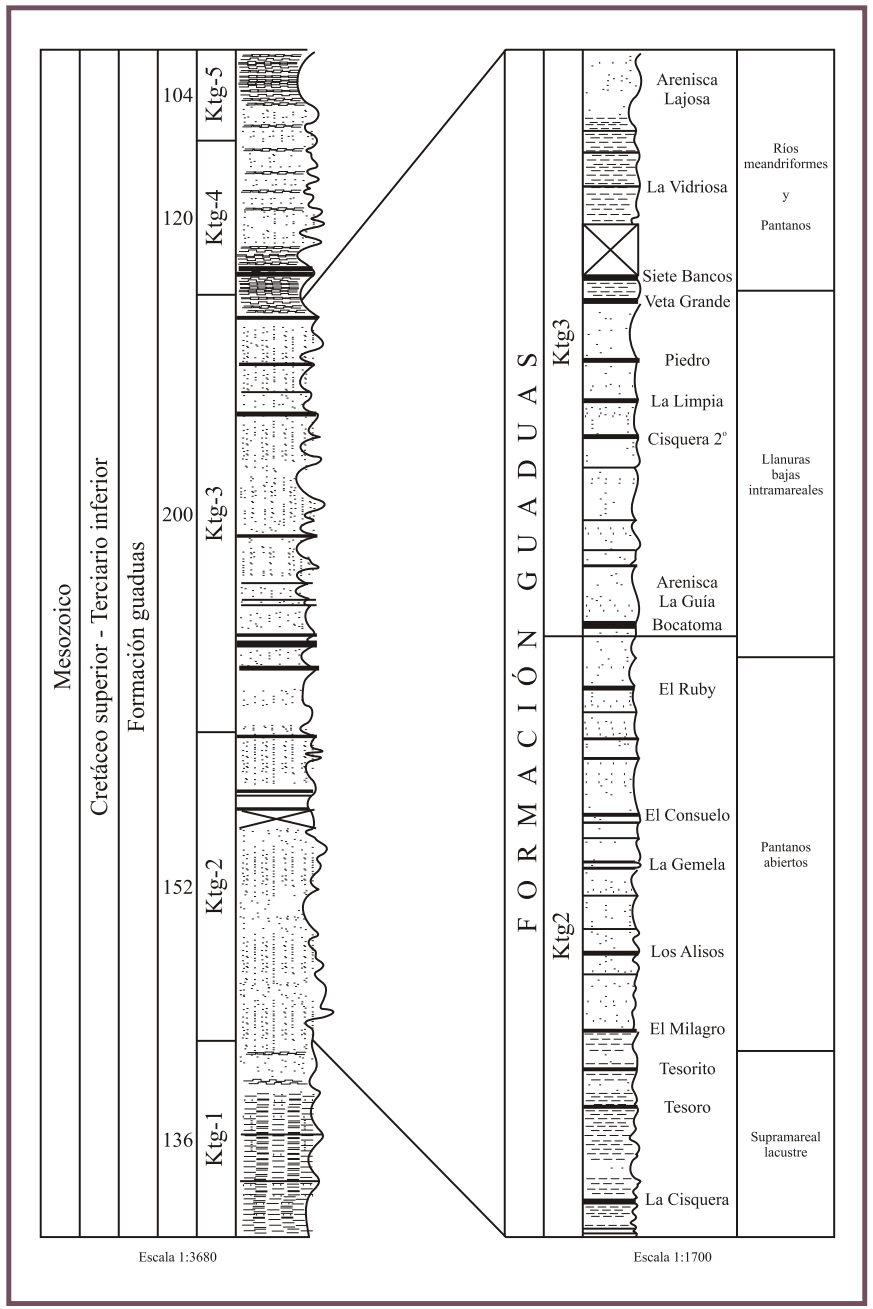

Figura 2. Columna estratigráfica Formación Guaduas, Samacá (Boyacá).

Tabla 1. Resultados de los análisis químicos para los carbones de Samacá, Boyacá, Colombia.

\begin{tabular}{|c|c|c|c|c|c|c|c|c|c|}
\hline Manto & $\begin{array}{l}\text { Humedad } \\
\text { Equilibrio }\end{array}$ & $\begin{array}{c}\text { Humedad } \\
\text { residual }\end{array}$ & Cenizas & $\begin{array}{c}\text { Materia } \\
\text { volátil }\end{array}$ & $\begin{array}{c}\text { Carbono } \\
\text { fijo }\end{array}$ & $\begin{array}{c}\text { Poder } \\
\text { calorífico }\end{array}$ & $\begin{array}{c}\text { Azufre } \\
\text { total }\end{array}$ & F.S.I. & Ambientes \\
\hline Veta grande & 3,7 & 1,3 & 11,1 & 30,8 & 54,3 & 6924 & 1,5 & 7 & \multirow{5}{*}{$\begin{array}{l}\text { Llanuras } \\
\text { bajas } \\
\text { intramareales }\end{array}$} \\
\hline Piedro & 1,0 & 1,1 & 9,8 & 31,4 & 57,6 & 7415 & 1,3 & 7,5 & \\
\hline La Limpia & 3,2 & 0,5 & 8,8 & 25,6 & 65 & 7993 & 0,8 & 8 & \\
\hline Cisquera $2 .^{\text {da }}$ & 3,2 & 0,8 & 11,4 & 29,2 & 56 & 7310 & 0,9 & 7,5 & \\
\hline Bocatoma & 2,1 & 0,7 & 5,7 & 27,2 & 66,4 & 7654 & 0,8 & 8 & \\
\hline Rubí & 2,7 & 1,1 & 3,3 & 24,9 & 70,7 & 8034 & 0,7 & 6 & \multirow{3}{*}{$\begin{array}{c}\text { Pantanos } \\
\text { abiertos }\end{array}$} \\
\hline Consuelo & 0,3 & 0,6 & 8,7 & 24,8 & 66 & 7840 & 0,6 & 8 & \\
\hline Gemela & 2,8 & 0,8 & 7,9 & 23,4 & 67,4 & 7554 & 0,4 & 8,5 & \\
\hline Tesorito & 2,6 & 0,8 & 3,8 & 19,6 & 76,4 & 8154 & 0,4 & 4 & \multirow{3}{*}{$\begin{array}{l}\text { Supramareal } \\
\text { lacustre }\end{array}$} \\
\hline Tesoro & 2,8 & 0,9 & 7,4 & 18,4 & 73,2 & 7523 & 0,3 & 7 & \\
\hline Cisquera & 8,9 & 0,8 & 7,1 & 18,8 & 73 & 7725 & 0,4 & 3,5 & \\
\hline
\end{tabular}


presentan acuñamientos, paleocanales y gradaciones de base a techo, con ambiente deposicional de canal de meandro.

En el nivel Ktg-4 se levantaron $120 \mathrm{~m}$ de arcillolitas grises y pardas con intercalaciones de lutitas negras carbonosas y dos cintas de carbón intercaladas con arcillolitas grises y pardas. La secuencia termina con $15 \mathrm{~m}$ de areniscas que corresponde a la Arenisca La Lajosa.

En el nivel Ktg-5 se levantaron $104 \mathrm{~m}$ de arcillolitas abigarradas (grises, rojas, amarillas, verdes) con intercalación de lutitas negras carbonosas y arenisca de grano fino. Ambientes de depósito de llanuras aluviales y canales meandriformes, estos últimos explican la presencia de paquetes de areniscas.

\section{RESULTADOS}

La figura 2 muestra la relación entre los ambientes de depósito, los mantos de carbón y los diferentes paquetes de roca que fueron depositados. En un proceso que comienza con ambientes mareales y lacustres, pasando por pantanos, para terminar con depósitos propios de llanuras aluviales.

La tabla 1 muestra los resultados de los análisis químicos para cada manto y su relación con los ambientes de depósito. Aunque la relación no es directa, se nota un aumento en la cantidad de ceniza a medida que el ambiente es más continental, como es el caso de los mantos Cisquera 2. ${ }^{\text {da }}$, Piedro y Veta grande; la misma tendencia se nota en el contenido de azufre (ver figuras 3 y 4 ).

La figura 5 muestra la relación entre el contenido de cenizas y el azufre total; la correspondencia entre cenizas y azufre total está dada por los materiales de origen singenético, ya que estos afectan la composición interna del carbón; hacia la parte derecha de la grafica la dispersión es representativa con valores superiores a los máximos admisibles para carbones coquizables.

El carbono fijo parece ser mayor en zonas mareales y pantanosas que en zonas de llanuras aluviales.

Las demás tendencias generales son conocidas, como son el aumento de cenizas y materia volátil a medida que los carbones se hacen más jóvenes y disminuye el carbono fijo y el poder calorífico.

El análisis de las figuras 6, 7 y 8 muestra la correlación de la materia volátil, poder reflectante de la vitrinita $(P R V / 2)$ y la vitrinita con respecto a la profundidad relativa de la columna estratigráfica de los niveles Ktg-2 y Ktg-3.

\section{DISCUSIÓN}

El propósito principal de esta investigación fue establecer los cambios de composición química con los cambios ambientales (ver figuras 6, 7 y 8 ).

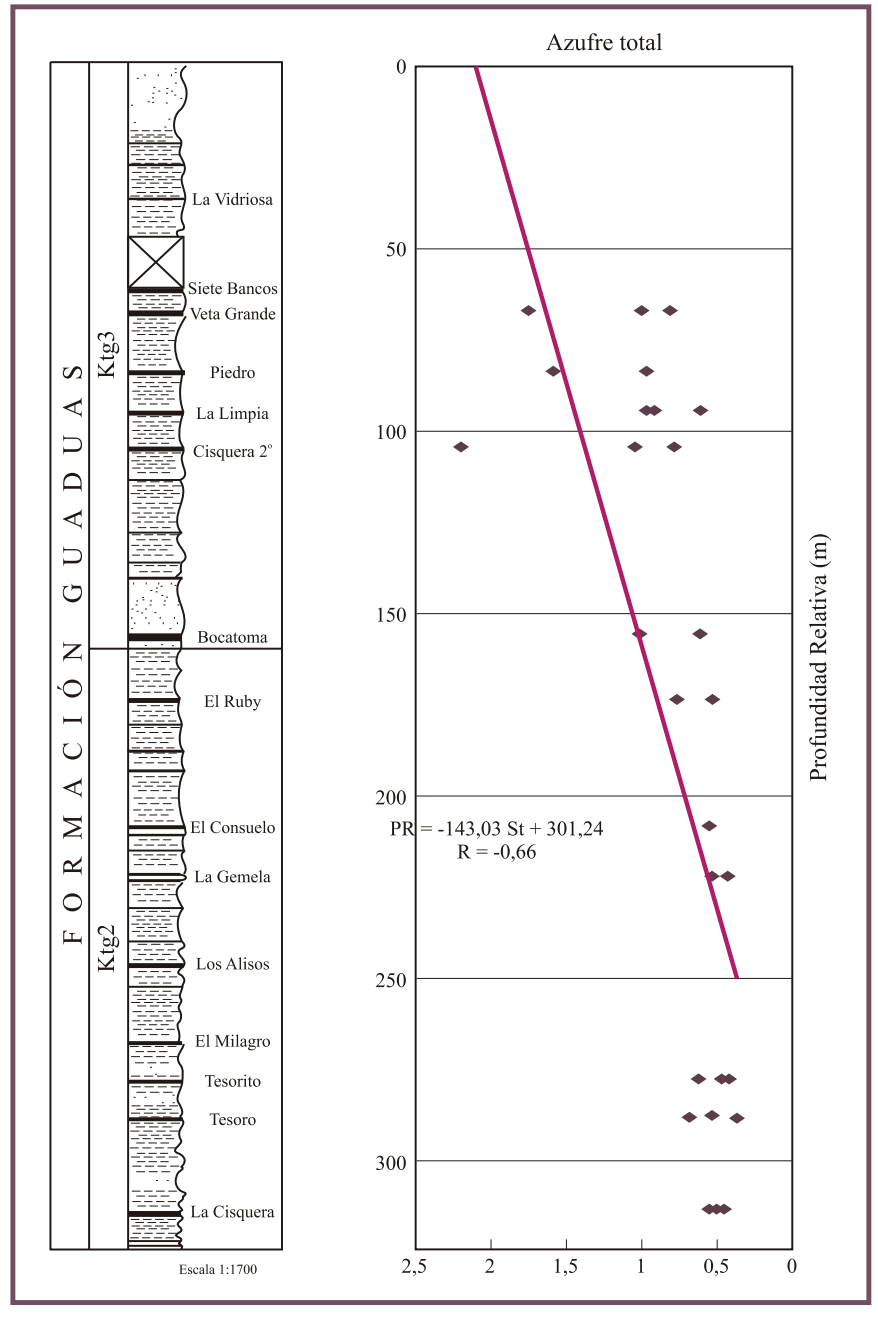

Figura 3. Correlación de azufre total y profundidad relativa.

Los mantos de carbón formados en un ambiente supramareal y lacustre presentan mínimos de materia volátil (ver figura 6), porque las diferentes facies (arenosa, arcillosa, orgánica) permiten la acumulación de niveles de arenisca por los cuales los gases del carbón (hidrógeno, nitrógeno, oxigeno) se desplazan a profundidades donde la presión es menor. Las arcillas acumuladas en ambientes de pantano abierto no permiten la inclusión de clastos dentro de la secuencia estratigráfica, por tanto, actúan como sellos, obstruyendo la movilidad de los gases, excepto cuando son alterados por eventos tectónicos. Las llanuras bajas intramareales presentan continuos cambios en la facie deposicional que permite la acumulación de gran cantidad de sedimentos, constituyendo mantos de carbón de espesor considerable, el bajo nivel del agua genera alta oxidación formando azufre pirítico en cristales euedrales, a diferencia de otros ambientes en los que solo se presentan trazas. Es importante destacar que gran parte del azufre total está constituido por azufre orgánico, producto de la acción de los agentes atmosféricos y cambios en el nivel freático. 


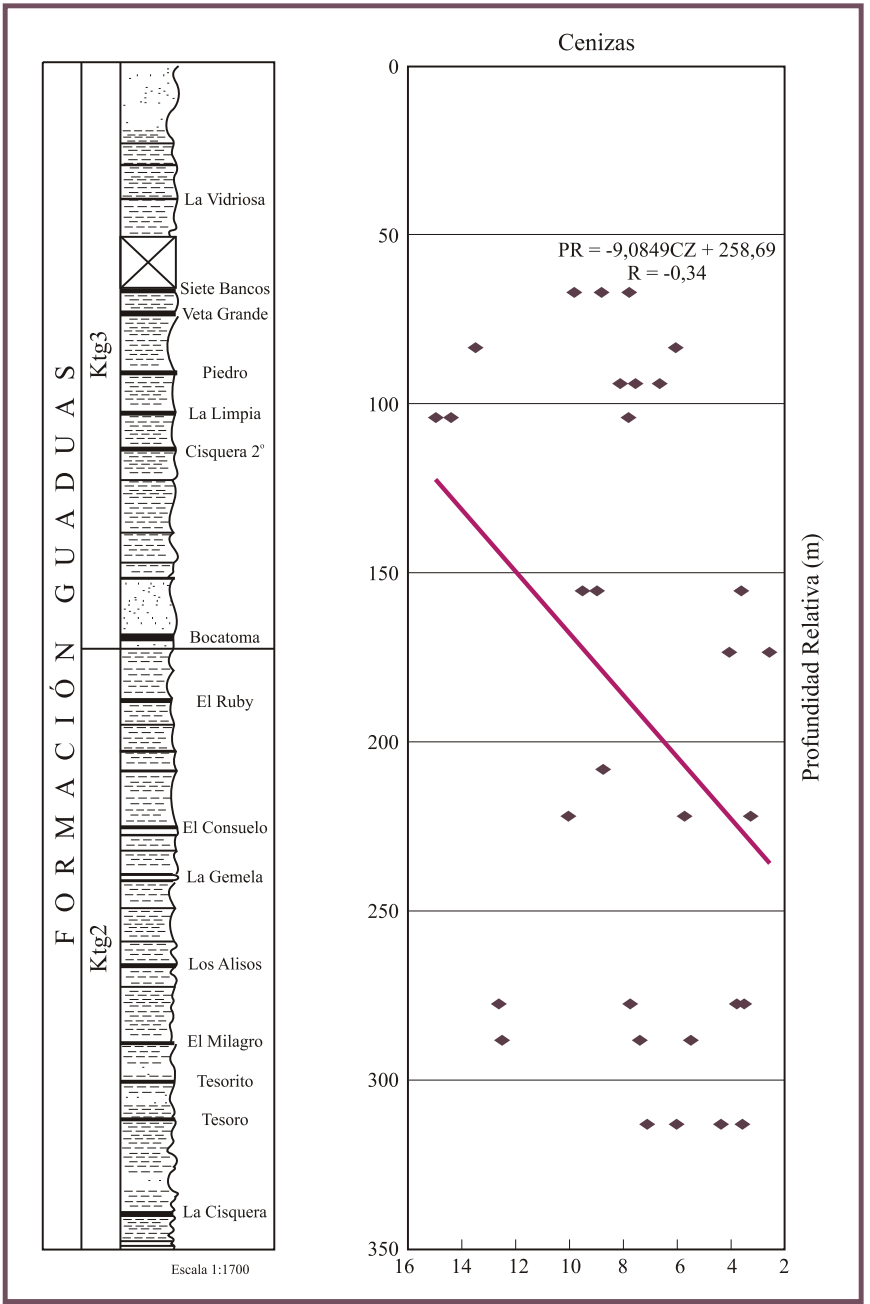

Figura 4. Correlación de cenizas

y profundidad relativa.

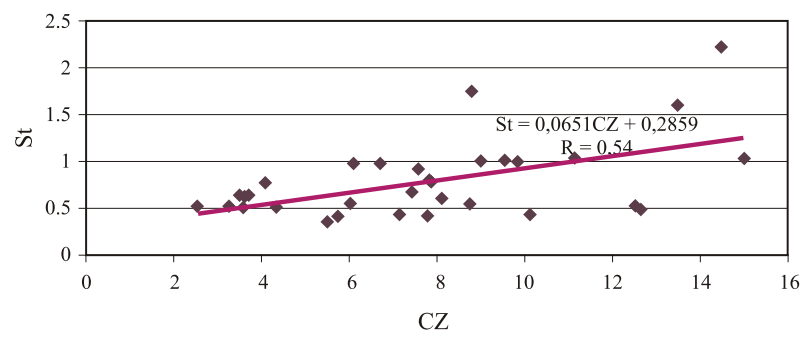

Figura 5. Relación entre el contenido

de cenizas y el azufre total.

A medida que el ambiente de depositación se hace más continental, disminuye el contenido de vitrinita y el poder de

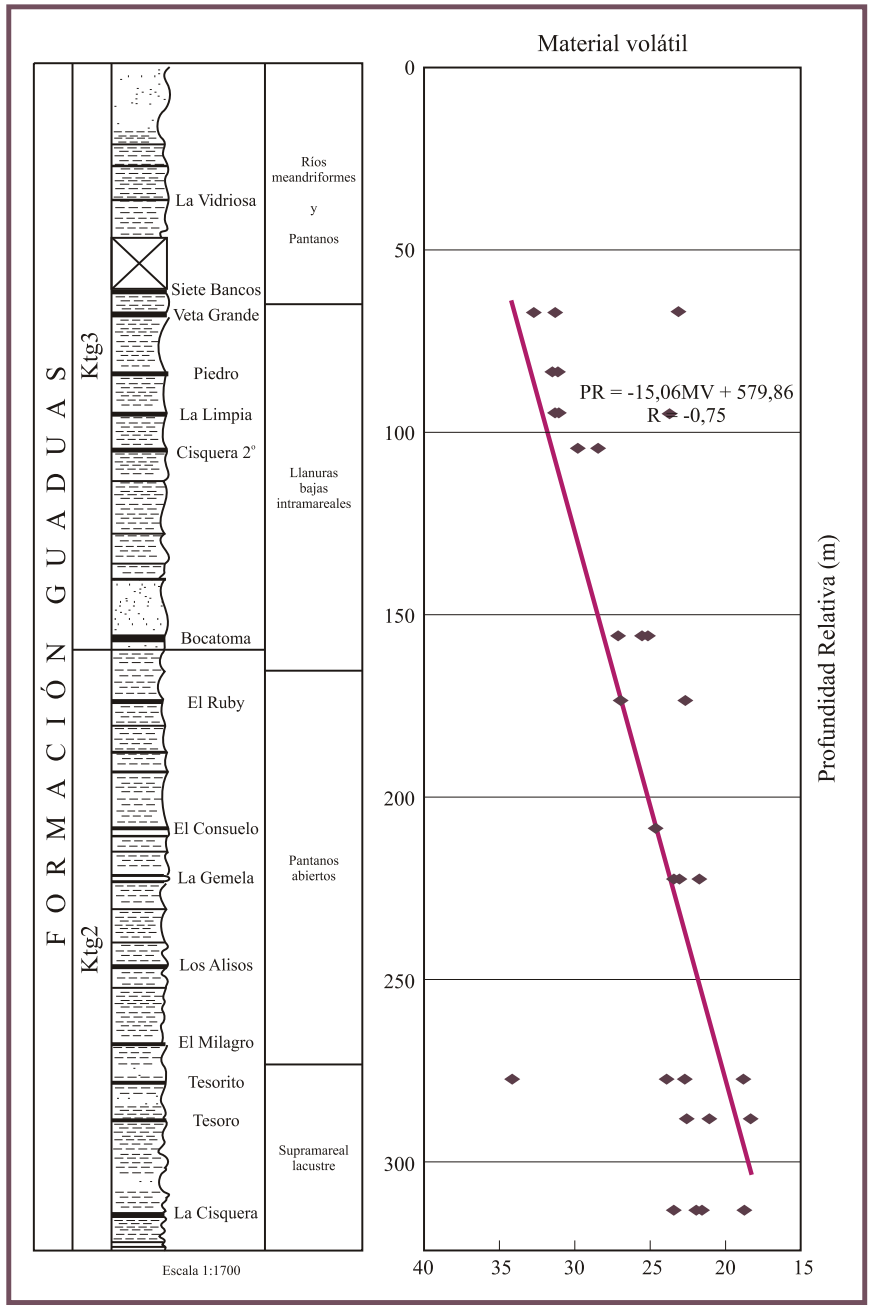

Figura 6. Correlación de materia volátil y profundidad relativa.

reflectancia (ver figuras 7 y 8). El carbono fijo, poder calorífico y FSI dependen directamente del comportamiento de la materia volátil, especialmente del contenido de hidrógeno.

Por último, los carbones formados en los ambientes supramareal, lacustre y de pantanos abiertos, combinados con la acción de los eventos tectónicos, presentan las características ideales para ser utilizados en la industria de coquización. Las llanuras bajas intramareales forman mantos de carbón con gran espesor, generadores de gas metano $\mathrm{CH}_{4}$, de gran interés en los programas de exploración gasífera.

Por razones económicas no se realizó el ensayo de dilatometría y no se incluyeron los resultados de reflectancia de la vitrinita.

Se sugiere adelantar un estudio sobre un mayor número de muestras que incluyan no solamente los ensayos realizados en el presente estudio, sino los resultados de otros estudios. 


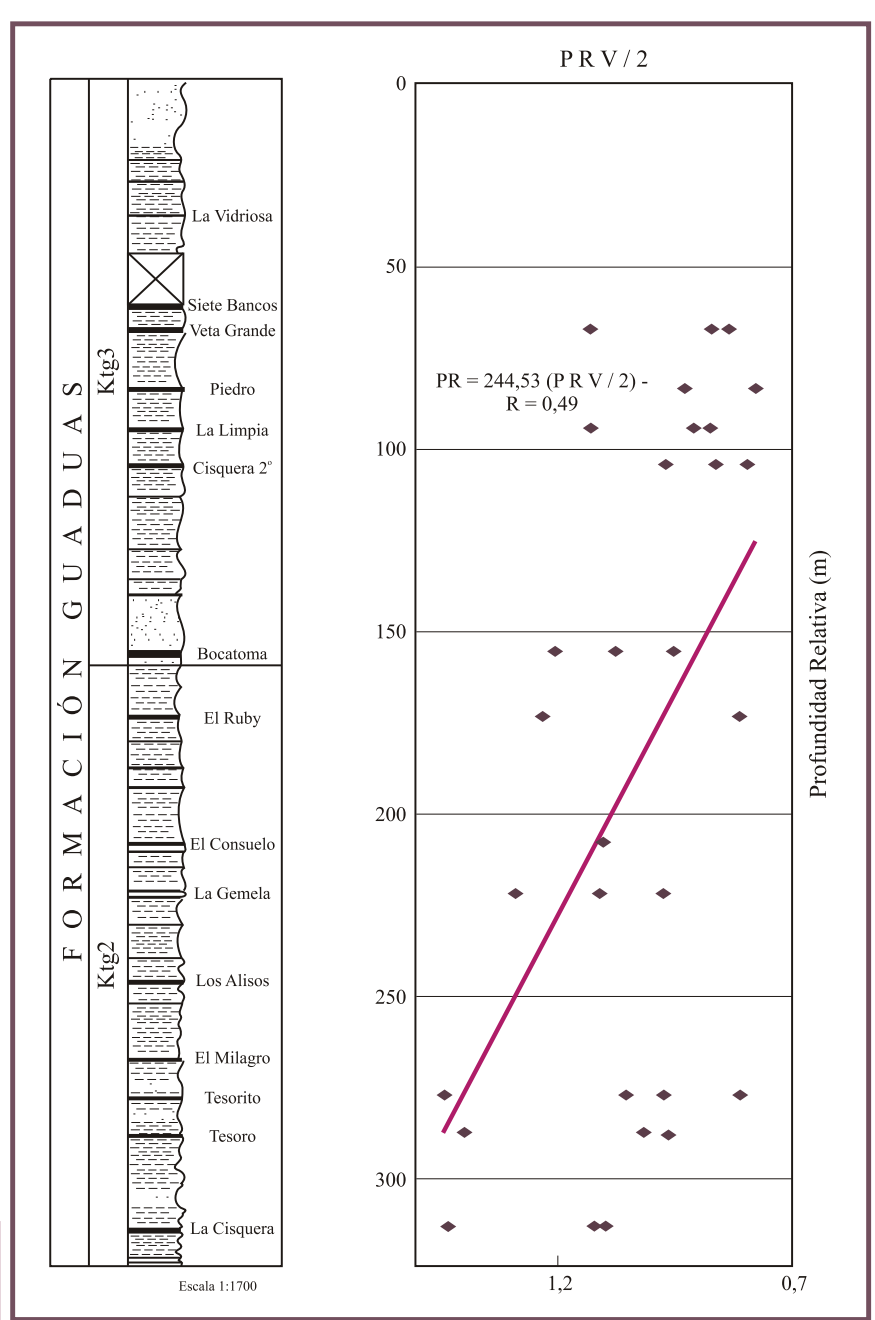

Figura 7. Correlación de PRV/2 y profundidad relativa.

\section{AGRADECIMIENTOS}

Esta investigación se desarrolló gracias al soporte financiero de la Comercializadora Colombiana del Carbón, Colcarbón, S.A. C.I., dentro del proyecto "Cartografía geológica, caracterización y cálculo de reservas para los carbones pertenecientes a los niveles Ktg-2 y Ktg-3 de la Formación Guaduas sobre el flanco occidental del Sinclinal ChecuaLenguazaque, entre los municipios de Ráquira y Samacá, Boyacá". Agradecimientos también al apoyo logístico recibido de la Universidad Pedagógica y Tecnológica de Colombia, Seccional Sogamoso.

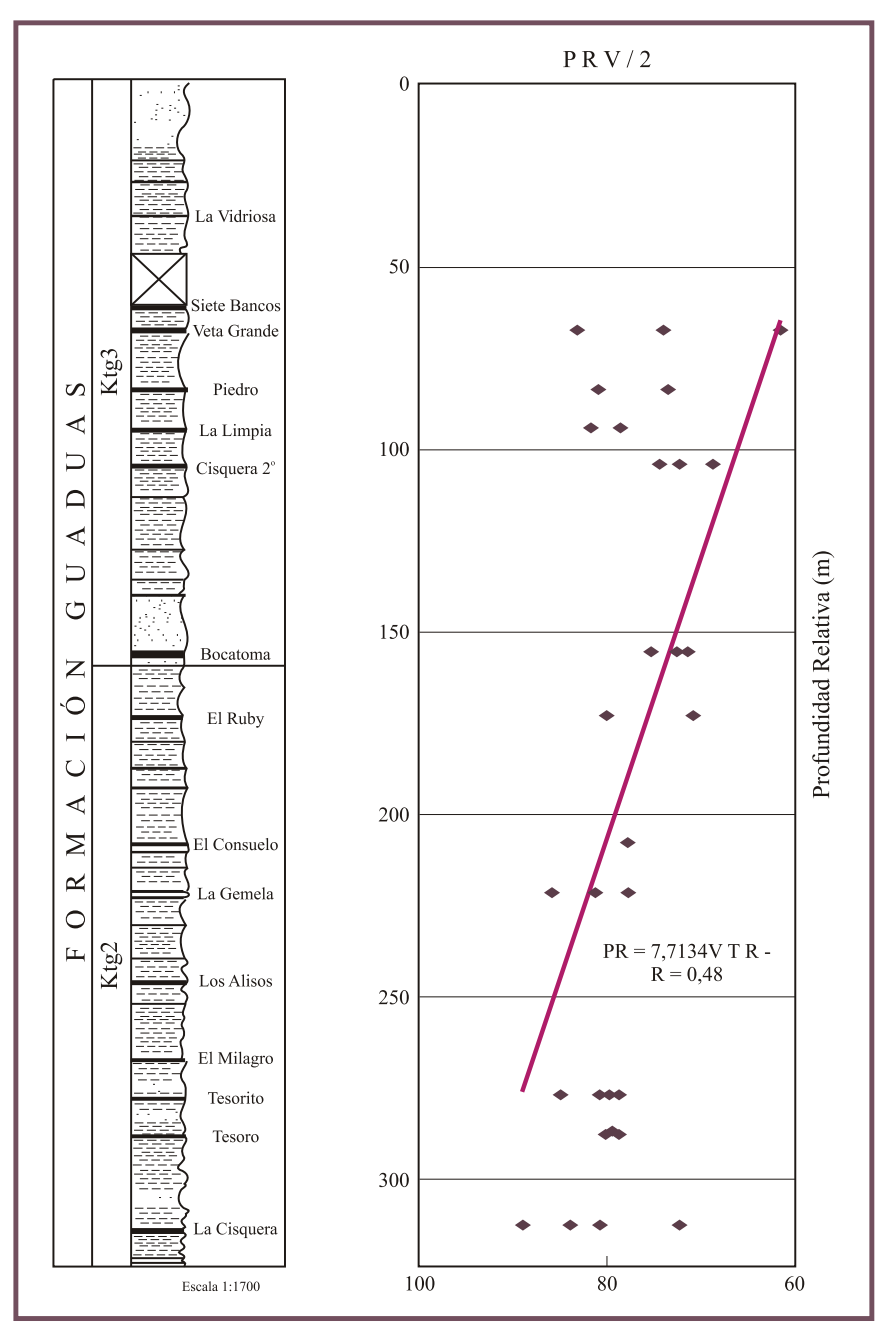

Figura 8. Correlación de vitrinita y profundidad relativa.

\section{REFERENCIAS}

Arboleda, C. (1987). Carbones coquizables del área Checua Samacá. Bogotá: Ingeominas.

ASTM (1999). Annual book ASTM Standards. USA.

Duarte, C. I. y Mariño, J. E. (2001). Utilización de las areniscas guías en la búsqueda y exploración de mantos de carbón. Caso Formación Guaduas. Sogamoso (Boyacá). V Congreso Nacional de Ciencia y Tecnología del Carbón. Valledupar, Cesar.

Hubach, E. (1957). Estratigrafía de la Sabana de Bogotá y alrededores. Bogotá: Ministerio de Minas, Boletín Geológico Vol. 5, Servicio Geológico Nacional,.

Pérez, F.; Valderrama, G.; Blanco, G.; Gonzáles, L.; García, F. (1987). Caracterización de carbones Colombianos, zona Checua Lenguazaque. Boletín Geológico Ingeominas, Vol. 28, N. ${ }^{\circ}$ 2, Bogotá.

Sarmiento P. (1992). Estratigrafía y medios de depósito de la Formación Guaduas. Boletín geológico, Vol. 32, Bogotá. 